\title{
Editorial \\ Lattice Computing: A Mathematical Modelling Paradigm for Cyber-Physical System Applications
}

\author{
Vassilis G. Kaburlasos (D)
}

check for

updates

Citation: Kaburlasos, V.G. Lattice Computing: A Mathematical Modelling Paradigm for Cyber-Physical System Applications. Mathematics 2022, 10, 271. https:// doi.org/10.3390/math10020271

Received: 2 January 2022

Accepted: 13 January 2022

Published: 16 January 2022

Publisher's Note: MDPI stays neutral with regard to jurisdictional claims in published maps and institutional affiliations.

Copyright: (C) 2022 by the author. Licensee MDPI, Basel, Switzerland. This article is an open access article distributed under the terms and conditions of the Creative Commons Attribution (CC BY) license (https:// creativecommons.org/licenses/by/ $4.0 /)$.
Human-Machines Interaction Laboratory (HUMAIN-Lab), Department of Computer Science, International Hellenic University (IHU), 65404 Kavala, Greece; vgkabs@teiemt.gr

By "model", we mean a mathematical description of a world aspect [1]. Mathematical models, implemented in software, are often engaged to drive hardware.

Models are typically developed in the Euclidean space $\mathrm{R}^{\mathrm{N}}$, for an integer number $\mathrm{N}$, where $\mathrm{R}$ is the totally ordered lattice of real numbers. Note that modelling in $\mathrm{R}^{\mathrm{N}}$ is inherently related to the physical world, where the conventional measurement process gives rise to the set, $\mathrm{R}$, of real numbers. Nevertheless, alternative spaces for modelling, even in physics, have been proposed, such as a probability space whose "event space" suggests a mathematical lattice. The interest here is in explicit, rigorous modelling in a mathematical lattice data domain. Mathematical lattices emerged in the mid-19th century as a spin-off of work on the formalizing of propositional logic [1]. Furthermore, it was Birkhoff's work in the mid-1930s [2] that started the general development of mathematical lattice theory.

The proliferation of computers has triggered a growing interest in mathematical lattices [3], including Boolean algebra for computer design as well as for software verification. As a result of the proliferation of computers, cyber-physical systems (CPSs) have emerged as devices with both sensing and reasoning capacities [4], which may also interact with humans. Notably, in addition to numerical (multimodal) sensory data during their interactions with one another, humans engage non-numerical data such as spoken language, gestures, rules, moral principles, concepts, symbols, etc. Therefore, for seamless interactions with humans, CPSs are also expected to cope with non-numerical data.

Lately, the "Lattice Computing (LC) information processing paradigm" has been proposed in CPS applications for mathematical modelling based on lattice theory by rigorously unifying (and fusing), in a Cartesian product, lattice-ordered numerical data and/or non-numerical data [4]. Especially promising is the capacity of mathematical lattices for knowledge representation [1]. For instance, mathematical lattices may suggest useful alternatives for representing human intensions/emotions and other.

Part of the interest of lattice theory is still in mathematics. However, the interest in practical applications is growing. This Special Issue presents recent advances in the LC paradigm, including studies in abstract/applied mathematics, formal concept analysis (FCA), mathematical morphology and image processing, machine learning, and computational intelligence, including both neural computing and fuzzy classifier designs. The proposed collection of studies includes eight articles outlined subsequently.

First, the abstract mathematics study [5] by Jia-Bao Liu, Mobeen Munir, Qurat-ul-Ain Munir and Abdul Rauf Nizami develops combinatorial invariants of some finite groups stemming from their corresponding lattice graphs. In conclusion, it calculates the diameter and girth, as well as other aspects of those lattice graphs.

Second, the machine learning study [6] by Chris Lytridis, Anna Lekova, Christos Bazinas, Michail Manios and Vassilis G. Kaburlasos introduces a k-nearest neighbor (kNN) classifier, namely, WINkNN, to time-series classification using lattice-ordered intervals' numbers (INs); the advantage of an IN is that it represents all-order data statistics in a time-series. Tunable, parametric non-linearities result in good performance in two 
benchmark datasets involving electroencephalography (EEG) signals, as well as audio time-series signals.

Third, the mathematical morphology study [7] by Marcos Eduardo Valle introduces the reduced dilation-erosion perceptron (r-DEP) classifier to deal with the shortage of a natural ordering of feature patterns by endowing the feature space with a reduced ordering. The proposed r-DEP classifier yielded average higher balanced accuracy scores than alternative classifiers from the literature on several binary classification datasets.

Additionally, the neural computing study [8] by Gerhard X. Ritter, Gonzalo Urcid and Luis-David Lara-Rodríguez presents a novel lattice-based biomimetic neural network trained by means of a similarity measure derived from a positive lattice valuation. Pattern recognition is achieved without any iterative scheme subject to convergence issues. Applications to real-world datasets demonstrate the overall classification performance.

Next, the applied mathematics study [9] by Francisco J. Valverde-Albacete and Carmen Peláez-Moreno introduces a basic technique for lattice computing, analogous to the singular value decomposition technique for rectangular matrices over complete idempotent semifields (i-SVD). It shows that instances of the proposed algebras are already useful in applications, e.g., morphological processing. A relation to an extension of formal concept analysis (FCA), namely K-FCA, is also shown.

Moreover, the applied mathematics investigation [10] by Francisco J. Valverde-Albacete and Carmen Peláez-Moreno extends the formal concept analysis (FCA) formalism to include all four Galois connections between four different semivectors spaces over idempotent semifields, resulting in K-four-fold formal concept analysis (K-4FCA). The partial results lead to a fundamental theorem that defines quadrilattices and discuss its relevance vis à vis previous formal conceptual analyses.

Furthermore, the machine-learning study [11] by Nina S. T. Hirata and George A. Papakostas firstly reviews and discusses the representational structure of morphological image operators, secondly addresses the problem of learning morphological image operators from data, and thirdly, it focuses on recent morphological image operator learning methods that take advantage of deep-learning frameworks.

Finally, the granular computing study [12] by Vassilis G. Kaburlasos, Chris Lytridis, Eleni Vrochidou, Christos Bazinas, George A. Papakostas, Anna Lekova, Omar Bouattane, Mohamed Youssfi and Takashi Hashimoto introduces the granule-based classifier (GbC) applicable to tree data structures, where a tree data structure represents a human face during human-robot interactions. Computational experiments, regarding three different pattern recognition problems, comparatively demonstrate the advantages of the $\mathrm{GbC}$ classifier.

Funding: This research received no external funding.

Acknowledgments: The research activity of the guest editor of this special issue has been carried out in the framework of the activities of the European Union's Horizon 2020 research and innovation program under the Marie Skłodowska-Curie grant agreement No 777720.

Conflicts of Interest: The author declares no conflict of interest.

\section{References}

1. Kaburlasos, V.G. Towards a Unified Modeling and Knowledge-Representation Based on Lattice Theory-Computational Intelligence and Soft Computing Applications; Series: Studies in Computational Intelligence; Springer: Heidelberg, Germany, 2006; Volume 27, ISBN 3-540-34169-2.

2. Birkhoff, G. Lattice Theory; American Mathematical Society, Colloquium Publications: Providence, RI, USA, 1967.

3. Ritter, G.X.; Urcid, G. Introduction to Lattice Algebra with Applications in AI, Pattern Recognition, Image Analysis, and Biomimetic Neural Networks; Chapman and Hall/CRC: Boca Raton, FL, USA, 2021; ISBN 9780367720292.

4. Kaburlasos, V.G. The Lattice Computing (LC) paradigm. In Proceedings of the 15th International Conference on Concept Lattices and their Applications (CLA 2020), Tallinn, Estonia, 29 June-1 July 2020; pp. 1-8. Available online: http://ceur-ws.org/Vol-2668/ (accessed on 5 January 2022).

5. Liu, J.-B.; Munir, M.; Munir, Q.-U.-A.; Nizami, A.R. Some Metrical Properties of Lattice Graphs of Finite Groups. Mathematics 2019, 7, 398. [CrossRef] 
6. Lytridis, C.; Lekova, A.; Bazinas, C.; Manios, M.; Kaburlasos, V.G. WINkNN: Windowed Intervals' Number kNN Classifier for Efficient Time-Series Applications. Mathematics 2020, 8, 413. [CrossRef]

7. Valle, M.E. Reduced Dilation-Erosion Perceptron for Binary Classification. Mathematics 2020, 8, 512. [CrossRef]

8. Ritter, G.X.; Urcid, G.; Lara-Rodríguez, L.-D. Similarity Measures for Learning in Lattice Based Biomimetic Neural Networks. Mathematics 2020, 8, 1439. [CrossRef]

9. Valverde-Albacete, F.J.; Peláez-Moreno, C. The Singular Value Decomposition over Completed Idempotent Semifields. Mathematics 2020, 8, 1577. [CrossRef]

10. Valverde-Albacete, F.J.; Peláez-Moreno, C. Four-Fold Formal Concept Analysis Based on Complete Idempotent Semifields. Mathematics 2021, 9, 173. [CrossRef]

11. Hirata, N.S.T.; Papakostas, G.A. On Machine-Learning Morphological Image Operators. Mathematics 2021, 9, 1854. [CrossRef]

12. Kaburlasos, V.G.; Lytridis, C.; Vrochidou, E.; Bazinas, C.; Papakostas, G.A.; Lekova, A.; Bouattane, O.; Youssfi, M.; Hashimoto, T. Granule-Based-Classifier (GbC): A Lattice Computing Scheme Applied on Tree Data Structures. Mathematics 2021, 9, 2889. [CrossRef] 